\title{
Role of Mycoplasma genitalium MG218 and MG317 cytoskeletal proteins in terminal organelle organization, gliding motility and cytadherence
}

\author{
Oscar O. Pich, Raul Burgos, Mario Ferrer-Navarro, Enrique Querol \\ and Jaume Piñol
}

Correspondence

Jaume Piñol

jaume.pinyol@uab.es

Received 19 May 2008

Revised 18 June 2008

Accepted 18 June 2008
Institut de Biotecnologia i Biomedicina and Departament de Bioquímica i Biologia Molecular, Universitat Autònoma de Barcelona, 08193 Bellaterra, Barcelona, Spain

\section{INTRODUCTION}

Mycoplasmas are genomically streamlined bacteria, characterized by the lack of a peptidoglycan layer and fastidious nutritional requirements. These parasitic micro-organisms have been continuously reemerging as important human and animal pathogens (Baseman \& Tully, 1997). In particular, Mycoplasma genitalium has been linked to numerous genitourinary as well as extragenitourinary human pathologies (Baseman et al., 1988) and it is a leading cause of Chlamydia -negative, non-gonococcal urethritis (Taylor-Robinson \& Horner, 2001; Tully et al., 1981). M. genitalium also has a unique and very attractive feature: with a genome size of $580 \mathrm{~kb}$ (Fraser et al., 1995), it is the smallest self-replicating cell known to be able to be grown in axenic culture. For this reason, this micro-organism has been considered to be one of the most suitable models for achieveing a complete understanding of the biology of a single replicating cell (Roberts, 2004).

M. genitalium cells display a differentiated tip structure, commonly known as the terminal organelle (TO), that

Abbreviations: EDC, electron-dense core; SAD, surface adherence defects; TB, terminal button; TO, terminal organelle.

A supplementary figure and table are available with the online version of this paper. gives them a flask-shaped appearance. The TO plays a key role in mycoplasma cytadherence and further parasitism of host target cells and it is defined by an electron-dense core (EDC) that is a component of the Triton X-100-insoluble fraction (Gobel et al., 1981; Meng \& Pfister, 1980). The intriguing complexity and the multifunctional role of the TO have attracted the interest of many researchers. Most of the cytoskeletal proteins present in the complex network that constitutes the TO have been identified primarily as cyadhesins or cytadherence-associated proteins. In addition, the TO is the leading end as mycoplasma cells glide (Radestock \& Bredt, 1977) and it constitutes the mycoplasma gliding motor (Hasselbring \& Krause, 2007). Motility and cytadherence are closely related in mycoplasmas and many cytadherence-related proteins are also required for cell gliding (Burgos et al., 2007; Hasselbring et al., 2005; Hasselbring \& Krause, 2007; Seto et al., 2005). However, the existence of a cytadherence-independent set of motility-related proteins has been disclosed recently for the slow gliding mycoplasmas M. genitalium (Pich et al., 2006a) and Mycoplasma pneumoniae (Hasselbring et al., 2006).

For several years the isolation and characterization of spontaneous as well as transposon-generated mutants involving different cytadherence-related genes has provided 
important information regarding the spatial and hierarchical development of the attachment organelle in $M$. pneumoniae (Krause \& Balish, 2004). These studies have indicated that the cytoskeletal protein HMW2 is a major structural element of the EDC (Balish et al., 2003b). In addition, it has been suggested that polymers of HMW3 protein surround the core and the terminal button (TB) in a linear pattern, possibly serving to stabilize this structure (Krause, 1996). Unfortunately, the specific contribution of the different cytoskeletal proteins from $M$. genitalium to the TO organization has been much less studied (Burgos et al., 2006, 2007). Two independent factors have probably hindered the study of TO formation and the whole cytadherence scenario in the smallest mycoplasma. First, screening for haemadsorption-negative colonies has not allowed the isolation of transposon-generated mutants involving any of the cytadherence-accessory genes from $M$. genitalium (Reddy et al., 1996). Second, the high rates of spontaneous occurrence of class I and class II haemadsorption-negative mutants (Mernaugh et al., 1993), which probably reflect a subjacent cytadherence phase variation mechanism (Burgos et al., 2006), hampers the screening process and jeopardizes the characterization of the mutants isolated.

In the present study, we aimed to investigate the involvement of $M$. genitalium cytoskeletal proteins in the TO organization and to analyse their contribution to gliding mechanics. To achieve this goal, a methodology recently developed in our laboratory (Pich et al., 2006a) was modified in order to isolate transposon-generated gliding-deficient mutants also displaying surface adherence defects (SAD). Several mutants showing transposon insertions within the $m g 218$ or $m g 317$ genes, encoding the orthologues of M. pneumoniae HMW2 and HMW3 cytadherence-accessory proteins, respectively, were isolated using this procedure. The analysis of the transposon mutants obtained has revealed that the reduced gliding motility and cytadherence exhibited by these mutants is well correlated with the presence of defects in their respective EDC, revealing the particular roles of MG218 and MG317 proteins in the TO architecture of $M$. genitalium.

\section{METHODS}

Culture conditions and transformation. M. genitalium strain G37 and the different mutants isolated in this study were grown in SP-4 broth (Tully et al., 1979) at $37{ }^{\circ} \mathrm{C}$ under $5 \% \mathrm{CO}_{2}$ in tissue culture flasks (TPP). For standard colony development, mycoplasma cells were plated in SP-4 medium supplemented with $0.8 \%$ agar (Difco). To check for gliding-deficient strains, cells were attached to cell culture dishes (Corning) and covered with SP-4 broth containing $0.5 \%$ low-melting-point agarose (Iberlabo). SP-4 medium was supplemented with tetracycline $\left(2 \mu \mathrm{g} \mathrm{ml} \mathrm{m}^{-1}\right.$; Roche) to select for resistant strains. Transformation of M. genitalium by electroporation was performed as described previously (Pich et al., 2006b).

DNA manipulation. Genomic DNA was isolated using the E.Z.N.A. Bacterial DNA kit (Omega Bio-tek). Sequencing of genomic DNA from the different transposon mutants obtained was performed with fluorescent dideoxynucleotides using the Big Dye 3.0 Terminator kit (Applied Biosystems) and Tc upstream and Tc downstream primers (Table 1), according to the recommendations of the manufacturer. Southern blot hybridization was performed using the Dig DNA labelling and detection kit (Roche).

Construction of plasmid $\mathbf{p} \Delta \mathbf{m g 2 1 8}$. To obtain plasmid $\mathrm{p} \Delta \mathrm{mg} 218$, the flanking regions of $m g 218$ were amplified by PCR. A $1 \mathrm{~kb}$ fragment corresponding to the upstream region of $m g 218$ was amplified using primers URmg218-5' and URmg218-3' (Table 1), cloned into an EcoRV-digested pBE plasmid (Pich et al., 2006b) and digested with PstI and EcoRI. Separately, a $0.8 \mathrm{~kb}$ fragment corresponding to the downstream region of $m g 218$ was amplified using primers DRmg218-5' and DRmg218-3' (Table 1), cloned into an EcoRV-digested pBE plasmid, and digested with BamHI and ApaI. Both flanking regions and an EcoRI-BamHI-digested tetM438 marker were finally cloned into a PstI-ApaI-digested pBSK plasmid to obtain the $\mathrm{p} \Delta \mathrm{mg} 218$ construct.

SDS-PAGE and MALDI-MS analysis. The separation of total mycoplasma proteins by SDS-PAGE followed standard procedures. The identification of MG317 protein in the SDS-PAGE was achieved by peptide mass fingerprint analysis, as described previously (Pich et al., 2006a). MG317 protein was identified with a score of 136 and a protein sequence coverage of $38.9 \%$. Scores greater than 68 were considered significant (Perkins et al., 1999).

Western blotting and preparation of antisera. Western blots were performed as described previously (Burgos et al., 2006), using MG218 monoclonal and polyclonal antibodies developed in this work, or rabbit antiserum to P41 (Krause et al., 1997), HMW1 (Stevens \& Krause, 1991) and HMW3 proteins (Stevens \& Krause, 1992). Monoclonal antibodies to MG218 protein were obtained by repeated

Table 1. Primers used in this work

\begin{tabular}{|ll|}
\hline Primer & \multicolumn{1}{c|}{ Sequence } \\
\hline Tc upstream & 5'-GGTAGTTTTTCCTGCATCAACATG-3' \\
Tc downstream & 5'-CGTCGTCCAAATAGTCGGATAG-3' \\
URmg218-5' & 5'-CTGCAGCCAGGATTTGATCCTAACCAAC-3' \\
URmg218-3' & 5'-GAATTCCATAAATTGGTTGCAGCGAAGG-3' \\
DRmg218-5' & 5'-GGATCCAACATTAAAAGGTTGATTGGCC-3' \\
DRmg218-3' & 5'-GGGCCCTAACAATTAACGGTGGTTCTTC-3' \\
mg218Exp5' & 5'-ACTGGTGGATCCCAACAAAAGCAAGATGAT-3' \\
mg218Exp3' & 5'-CAGGTACTCGAGTTTACTTGCTGCTTTTTG-3' \\
\hline
\end{tabular}


immunization of BALB/c mice with a M. genitalium $0.1 \%$ Triton X100 -insoluble fraction in sterile PBS. Polyclonal antibodies to the MG218 C-terminal region were obtained by repeated immunization of BALB/c mice with a purified, recombinant C-terminal MG218 polypeptide (amino acids 1627-1805). Previously, a 537 bp sequence corresponding to nucleotides 264055-264592 of the M. genitalium genome was amplified by PCR using primers mg218Exp5' and mg218Exp3' (Table 1), cloned into a pET21a vector (Novagen), expressed in Escherichia coli BL21(DE3) and purified following standard procedures.

Microcinematography. Gliding motility data for the wild-type strain and the different mutants was obtained from microcinematographic studies (Pich et al., 2006a). The motility of 25 individual cells from each particular clone of $\mathrm{mg} 317^{-}$or $\mathrm{mg} 218^{-}$mutants was analysed. Data obtained from the different $\mathrm{mg} 317^{-}$mutant clones or, separately, from the different $\mathrm{mg} 218^{-}$mutant clones, were combined to obtain a single value. Similarly, a total of 300 cells from $\mathrm{mg} 317^{-}$or mg218 ${ }^{-}$mutants, including cells from different clones, were examined to calculate the respective gliding frequencies.

Haemadsorption activity. For quantitative assessment of the haemadsorption activity, aliquots of $25 \mu$ l containing $\sim 10^{7}$ c.f.u. $\mathrm{ml}^{-1}$ of the wild-type strain, mutants $c 9, c 5, c 12, c 6, c 4$ and c16, and a representative clone of $\Delta \mathrm{mg} 218$ and $\Delta \mathrm{mg} 191$ mutants (Burgos et al., 2006), were incubated at $37{ }^{\circ} \mathrm{C}$ for $2 \mathrm{~h}$ with $475 \mu \mathrm{l}$ diluted $(1: 50)$ human erythrocytes. The mixture was shaken gently throughout the incubation. Erythrocytes and bound mycoplasmas were pelleted by centrifugation at $300 g$ for $2 \mathrm{~min}$. Different dilutions of the supernatant were plated onto solid SP-4 medium in order to estimate the mycoplasma concentration. Prior to the incubation with erythrocytes, different dilutions of the original samples were also plated onto solid SP-4 medium to determine the initial mycoplasma concentration. The haemadsorption activity values are derived from two independent experiments. In each experiment, data obtained from the two clones representing the same group of mutants were combined.

Scanning electron microscopy. Scanning electron microscopy analyses were performed using Permanox chamber slides (Nunc). For this purpose, cells from the wild-type strain, all $\mathrm{mg} 317^{-}$mutants, and mg218 ${ }^{-}$mutants c12, c13, c11, c3 and c6 were grown for $24 \mathrm{~h}$, while cells from $\Delta \mathrm{mg} 218$ mutants and mg218 ${ }^{-}$mutants $\mathrm{c} 4$ and $\mathrm{c} 16$ were grown for $48 \mathrm{~h}$. SP-4 medium was removed and cells were washed gently with PBS and fixed with $1 \%$ glutaraldehyde for $1 \mathrm{~h}$. Samples were washed again with PBS and dehydrated sequentially with 25, 50, 75 and $100 \%$ ethanol concentrations for periods of 10 min each. Immediately, samples were critical-point dried (K850 Critical Point Drier, Emitech) and sputter coated with gold. Samples were observed using a Hitachi S-570 scanning electron microscope.

Transmission electron microscopy. Cells from the wild-type strain, $\mathrm{mg} 218^{-}$mutants $\mathrm{c} 4, \mathrm{cl} 6$ and $\mathrm{c} 6, \mathrm{mg} 317^{-}$mutant $\mathrm{c5}$ and $\Delta \mathrm{mg} 218$ mutant c8 were grown to late-exponential phase, scraped, pelleted by centrifugation, and washed with PBS. Pellets were fixed with $2.5 \%(\mathrm{v} / \mathrm{v})$ glutaraldehyde and $2 \%(\mathrm{v} / \mathrm{v})$ paraformaldehyde (EM grade, TAAB) in $100 \mathrm{mM}$ phosphate buffer (PB, pH 7.4) for $2 \mathrm{~h}$ and rinsed with $100 \mathrm{mM}$ PB. Pellets were then post-fixed in $1 \%(\mathrm{w} / \mathrm{v})$ osmium tetroxide (TAAB) containing $0.8 \%(\mathrm{w} / \mathrm{v})$ potassium hexacyanoferrate (III) (Sigma) for $2 \mathrm{~h}$ and washed with $100 \mathrm{mM}$ PB. All these steps were done at $4{ }^{\circ} \mathrm{C}$. Samples were dehydrated through a graded ethanol series, infiltrated in Spurr's resin and polymerized for $48 \mathrm{~h}$ at $60{ }^{\circ} \mathrm{C}$. Ultrathin sections were mounted on copper grids, contrasted with standard uranyl acetate and lead citrate double-staining, and observed in a Hitachi H-7000 transmission electron microscope at $75 \mathrm{kV}$. The length of the EDC in a particular strain was determined from measurements of 18-25 TO.

\section{RESULTS}

\section{Isolation of M. genitalium gliding-deficient mutants with SAD}

A random transposon library was obtained by electroporating wild-type cells in the presence of MTnTetM438, a modified version of $\mathrm{Tn} 4001$ that confers tetracycline resistance and gives rise to random and stable insertions in the M. genitalium genome (Pich et al., 2006b). Transformed cells were dispensed into cell culture dishes, left to adsorb onto the plastic surface and covered with $0.5 \%$ low-melting-point agarose SP-4 medium. In contrast to our previous report (Pich et al., 2006a), the adsorbed cells were not washed with PBS, in order to retain those mutants not strongly adhered to the plastic surface. The morphology of $\sim 12000$ colonies was examined and 40 colonies exhibiting a compact morphology, compatible with the existence of gliding deficiencies, were identified and isolated. Since gliding-deficient mutants without SAD are also isolated by this procedure, we first discarded those mutants showing transposon insertions in the $m g 200$ and $m g 386$ genes, analysed extensively in our previous report. Twenty-two transposon mutants lacking MG386 or MG200 polypeptides were identified by SDS-PAGE analysis and consequently were not examined. Separately, seven clones containing mixed populations were also discarded.

\section{Identification of the transposon insertion points}

Insertion points of MTnTetM438 in the eleven glidingdeficient mutants selected for further analysis (Table 2) were determined by sequencing the corresponding genomic DNA using the Tc upstream and Tc downstream primers (Table 1) present in the MTnTetM438 transposon. Seven transposon mutants showed MTnTetM438 insertions within the $m g 218$ coding region (mg218 ${ }^{-}$mutants; Fig. 1a). The remaining transposon mutants showed minitransposon insertions within the $m g 317$ coding region (mg317 ${ }^{-}$mutants; Fig. 1b).

\section{Colony morphology of the mutants}

Cells from mg $218^{-}$mutants c4 and c16 covered with $0.5 \%$ low-melting-point agarose SP-4 medium developed flat and shiny colonies, with several small colonies surrounding the main one (Fig. 2a). Interestingly, cells from both clones did not attach avidly to the plastic surface of the cell culture flask. In contrast, under similar culture conditions, cells from the remaining $\mathrm{mg} 218^{-}$mutant clones (Fig. 2b) and all the $\mathrm{mg} 317^{-}$mutants (Fig. 2c) developed colonies with rough surfaces and a granulate appearance in the centre. The presence of some satellite microcolonies in these clones indicated that a certain degree of locomotion was still preserved. Cells from these nine transposon mutants attached quite well to the plastic surface of cell culture flasks. Hence, the two different classes of colony morphologies described here reflect the effect of the gene disruption 
Table 2. MTnTetM438 insertion point in the 11 gliding deficient mutants

\begin{tabular}{|c|c|c|c|c|c|}
\hline \multirow[t]{4}{*}{$m g 218$} & $c 4$ & $259188(-)$ & 3 & - & - \\
\hline & c12 & $260913(+)$ & 577 & 69.0 & \pm \\
\hline & c13 & $261124(+)$ & 648 & 77.7 & \pm \\
\hline & c6 & $261575(+)$ & 799 & 95.3 & \pm \\
\hline \multirow[t]{4}{*}{$m g 317$} & $\mathrm{cl}$ & $396783(-)$ & 213 & 24.4 & \pm \\
\hline & c9 & $396712(+)$ & 237 & 26.9 & \pm \\
\hline & c5 & $396582(-)$ & 280 & 31.8 & \pm \\
\hline & c15 & $396348(-)$ & 358 & 40.7 & \pm \\
\hline
\end{tabular}

*The insertion point refers to the NC 000908 GenBank genome sequence from M. genitalium. Symbols (+) [plus strand] and (-) [minus strand] indicate the sense of the MTnTetM438 insertions in the genome.

$\dagger \pm$, Presence of moderate cell adherence defects; -, presence of severe cell adherence defects.

on the surface-adherence capability of the mutant strain. This important divergence prompted us to consider the separation of the mg2 $18^{-}$mutants into two groups: $\mathrm{mg} 218^{-}$ mutants with severe SAD (c4 and c16), and mg218 mutants with moderate SAD (c3, c6, c11, c12 and c13).

\section{Construction of $\Delta \mathrm{mg} 218$ mutants}

In the light of the two different phenotypes observed after transposon disruption of $m g 218$, we decided to analyse the effect of the complete loss of MG218 protein in $M$. genitalium by deleting the $m g 218$ coding region completely by homologous recombination. For this purpose, the suicide plasmid $\mathrm{p} \Delta \mathrm{mg} 218$ was engineered to contain the tetM438 selectable marker (Pich et al., 2006b) and the flanking regions of $m g 218$ (Fig. 3a). A double recombination event between plasmid p $\Delta \mathrm{mg} 218$ and the mycoplasma genome promotes the substitution of bases 43 to 5376 of

(a) mg218' mutants

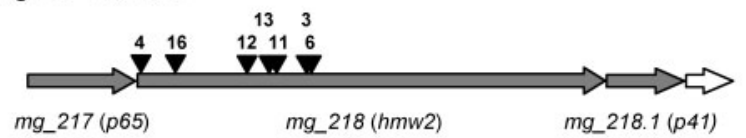

(b) mg317- mutants

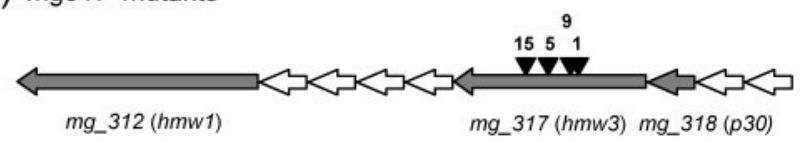

Fig. 1. Schematic representation showing the respective locations of MTnTetM438 insertions within the $m g 218$ (a) and $m g 317$ genes (b). Full arrows represent the genes for which the locus_tag is shown. Gene names in parentheses correspond to the respective $M$. pneumoniae homologues. $m g 218.1$ has also been annotated as $m g 491$. $m g 218$ (98.5\% of the $m g 218$ coding region) by the tetM438 marker. Several tetracycline-resistant clones were isolated after electroporation of $M$. genitalium in the presence of $\mathrm{p} \Delta \mathrm{mg} 218$. The presence of the expected deletion within $m g 218$ in these strains was assessed by Southern blotting. Genomic DNA from the wild-type strain and eight $\mathrm{p} \Delta \mathrm{mg} 218$ transformants was digested with Acc65I and probed with a $0.8 \mathrm{~kb} B a m \mathrm{HI}-A p a \mathrm{I}$ fragment obtained from plasmid $\mathrm{p} \Delta \mathrm{mg} 218$. A single band of $2.2 \mathrm{~kb}$, clearly different from the $10.9 \mathrm{~kb}$ band expected for the wild-type strain, was detected for the eight $\mathrm{p} \Delta \mathrm{mg} 218$ transformants analysed (Fig. 3b). This single band of $2.2 \mathrm{~kb}$ is compatible only with a double recombination event and confirms the expected deletion of $m g 218$ in these strains. Hereinafter, we will refer to these strains as $\Delta \mathrm{mg} 218$ mutants.

\section{Gliding motility data}

Gliding motility of the wild-type strain and $\mathrm{mg} 218^{-}$, mg317 ${ }^{-}$and $\Delta \mathrm{mg} 218$ mutants was analysed quantitatively by microcinematography (Table 3 ). Motile cells from the wild-type strain represented $93.8 \%$ of the cell population and exhibited a mean gliding speed of $0.152 \mu \mathrm{m} \mathrm{s}{ }^{-1}$. However, attached cells from $\mathrm{mg} 17^{-}$and $\mathrm{mg} 218^{-}$ mutants with moderate SAD displayed a mean gliding speed between four and fivefold below that of the wild-type strain. Motile cells from $\mathrm{mg}^{-} 7^{-}$mutants represented $48.3 \%$ of the cell population while the percentage of motile cells from mg218 ${ }^{-}$mutants with moderate SAD was slightly lower (43.9\%). In contrast, $\Delta \mathrm{mg} 218$ and mg218 mutants with severe SAD were non-motile.

In addition, numerous cells from $\mathrm{mg} 218^{-}$and $\mathrm{mg} 317^{-}$ mutants displayed filaments (see below: Cell morphology) similar to those documented previously for $m g 386$ glidingdeficient mutants (Pich et al., 2006a). These filaments were often found connecting two different cells but were also 

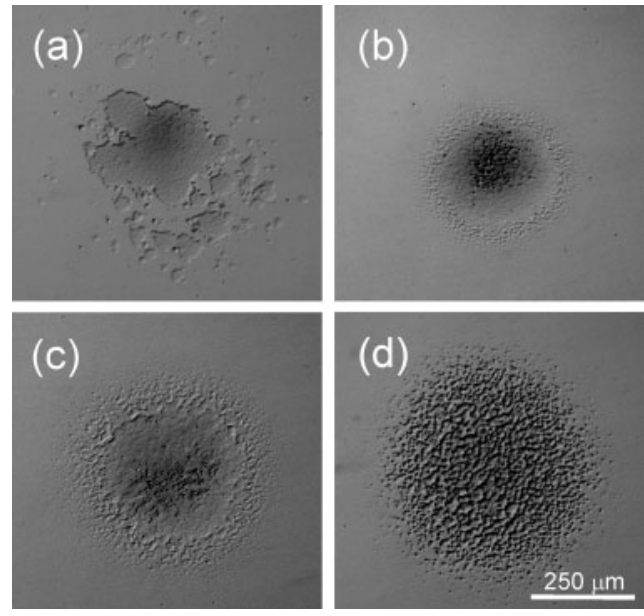

Fig. 2. Colony morphology of mycoplasma cells attached to cell culture dishes and covered with SP-4 medium containing $0.5 \%$

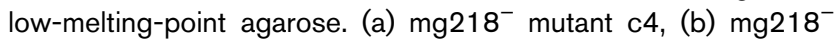
mutant c6, (c) mg317 ${ }^{-}$mutant c5, (d) wild-type strain.

present in isolated mycoplasmas. We observed that the filament linking two cells was broken as the cells became further apart (data not shown). When the resulting individual cells resumed gliding, the filament was clearly detected at the pole opposite to the direction of movement.

\section{Protein profile}

The protein profiles of the different mutants isolated in this study were analysed by SDS-PAGE (Fig. 4a) and Western blotting (Fig. 4b-f). As expected, a $220 \mathrm{kDa}$ band corresponding to full-length (1805 amino acids) MG218 protein (Dhandayuthapani et al., 1999) was absent in both $\Delta \mathrm{mg} 218$ and mg218 ${ }^{-}$mutants (Fig. 4a, b). Nevertheless, the use of MG218 monoclonal antibodies revealed the presence of N-terminal MG218 derivatives in all mg218 mutants with moderate SAD (Fig. 4b). Polyclonal antibodies raised against amino acids 1627 to 1805 of MG218 protein revealed also the presence of several C-terminal
MG218 derivatives (bands of 118, 115 and $25 \mathrm{kDa}$, Fig. 4d), most of them visible both in the wild-type strain and in all the mg $218^{-}$mutants isolated. As expected, no N-terminal and C-terminal MG218 derivatives were observed among the $\Delta \mathrm{mg} 218$ mutants.

All $\mathrm{mg} 317^{-}$mutants lacked the band corresponding to fulllength (599 amino acids) MG317 protein (Svenstrup et al., 2006). However, the presence of truncated MG317 fragments was not detected in this case. $\Delta \mathrm{mg} 218$ and $\mathrm{mg} 218^{-}$ mutants c16 and c12 (Fig. 4a, c) as well as mutant c4 (data not shown) also exhibited reduced levels of MG317. Likewise, levels of MG312 protein, the orthologue of $M$. pneumoniae HMW1, were also reduced in $\Delta \mathrm{mg} 218$ and mg218 ${ }^{-}$mutants $\mathrm{c} 4, \mathrm{c} 16$ and $\mathrm{cl} 2$ (Fig. 4e). These observations strongly suggest that the stability of MG312 and MG317 depends on the expression of the MG218 Nterminal region, in particular of the short stretch located between amino acids 577 and 648 (the length of the Nterminal MG218 derivatives in mutants c12 and c13). In addition, all the mutants isolated in this study showed reduced levels of MG386, P140 and P110 proteins. The stability of MG386 protein seems to be directly linked to the expression of P140 and P110 proteins at wild-type levels (Burgos et al., 2006), suggesting a cooperative interaction between these proteins. Finally, the presence of MG218.1 protein, the orthologue of $M$. pneumoniae $\mathrm{P} 41$, was detected in all $\Delta \mathrm{mg} 218$ and $\mathrm{mg} 218^{-}$mutants (Fig. 4f). This result rules out the existence of polar effects on the $m g 218.1$ gene derived from disruption or deletion of $m g 218$. Polar effects derived from the transposon insertion in $\mathrm{mg} 317^{-}$mutants are not expected because the distance between $m g 316$ and $m g 317$ (40 bp) is too long to allow translational coupling of the corresponding polypeptides (Lluch-Senar et al., 2007).

\section{Haemadsorption activity}

Haemadsorption activity of the different mutants isolated in this study, the wild-type strain and the non-adherent mutant $\Delta \mathrm{mg} 191$ was analysed quantitatively. As expected, mg317 ${ }^{-}$and $\mathrm{mg} 218^{-}$mutants with moderate SAD retained around $50 \%$ of the wild-type haemadsorption (a)

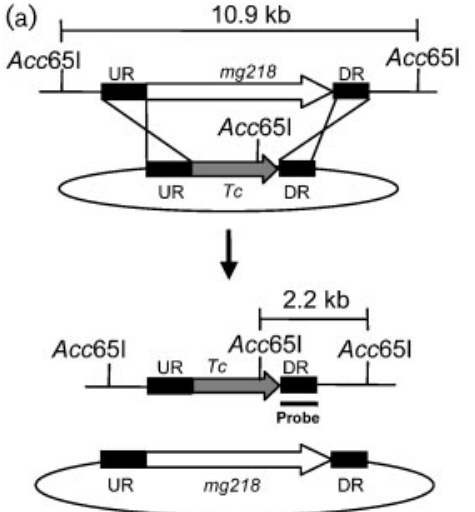

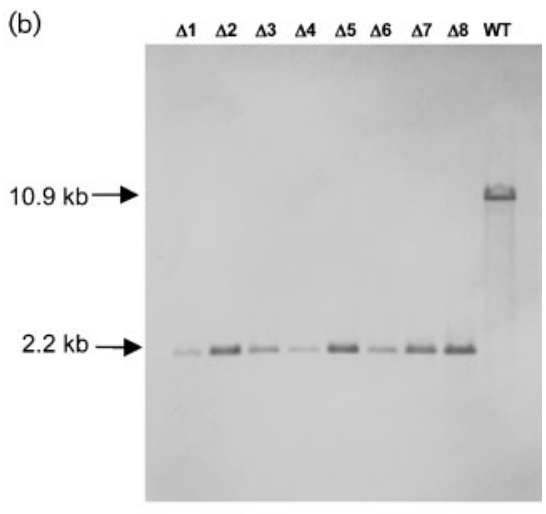

Fig. 3. (a) Schematic representation showing the genomic rearrangements when obtaining $\Delta$ mg218 mutants and (b) Southern blot analysis of these mutants. UR, upstream region; DR, downstream region; WT, wildtype. 
Table 3. Motility and cytadherence data

ND, Not determined; HA, haemadsorption; WT, wild-type.

\begin{tabular}{|c|c|c|c|c|}
\hline Strain & $\begin{array}{l}\text { Mean gliding speed } \\
\left(\mu \mathrm{m} \mathrm{s}^{-1}\right) \pm \mathrm{SEM}\end{array}$ & $\begin{array}{l}\text { Mean gliding speed } \\
\left.\text { ranges }^{\star}(\mu \mathrm{m} \mathrm{s})^{-1}\right)\end{array}$ & Motile cells & $\begin{array}{l}\text { HA activity } \\
(\% \text { of WT) }\end{array}$ \\
\hline WT & $0.152 \pm 0.007$ & - & $93.8 \%$ & 100 \\
\hline $\operatorname{mg} 218^{-}$mutants $\dagger$ & $0.034 \pm 0.004$ & $0.033-0.037$ & $43.9 \%$ & 46.5 \\
\hline $\operatorname{mg} 317^{-}$mutants & $0.032 \pm 0.004$ & $0.031-0.034$ & $48.3 \%$ & 62.4 \\
\hline$\Delta \mathrm{mg} 218$ mutants & 0 & - & $0 \%$ & 19.3 \\
\hline$\Delta$ mg191 mutants & $\mathrm{ND}$ & - & ND & 10.8 \\
\hline
\end{tabular}

${ }^{\star}$ Maximum and minimum mean gliding speed values obtained for the different clones.

$\nmid$ Clones with moderate SAD only.

activity (Table 3). Accordingly, cells from these mutants grow mainly attached to the plastic surface of the cell culture flask in liquid cultures. In contrast, $\Delta \mathrm{mg} 218$ mutants displayed haemadsorption activities $20 \%$ of that of the wild-type strain. Correspondingly, cells from $\Delta \mathrm{mg} 218$ mutants grow mainly in suspension in liquid cultures. The
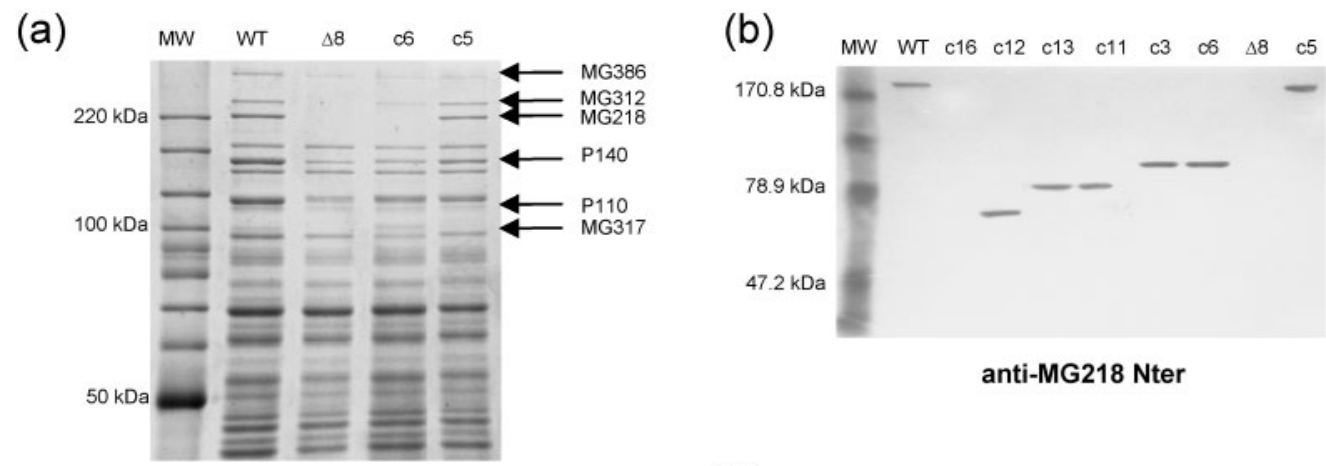

(d)
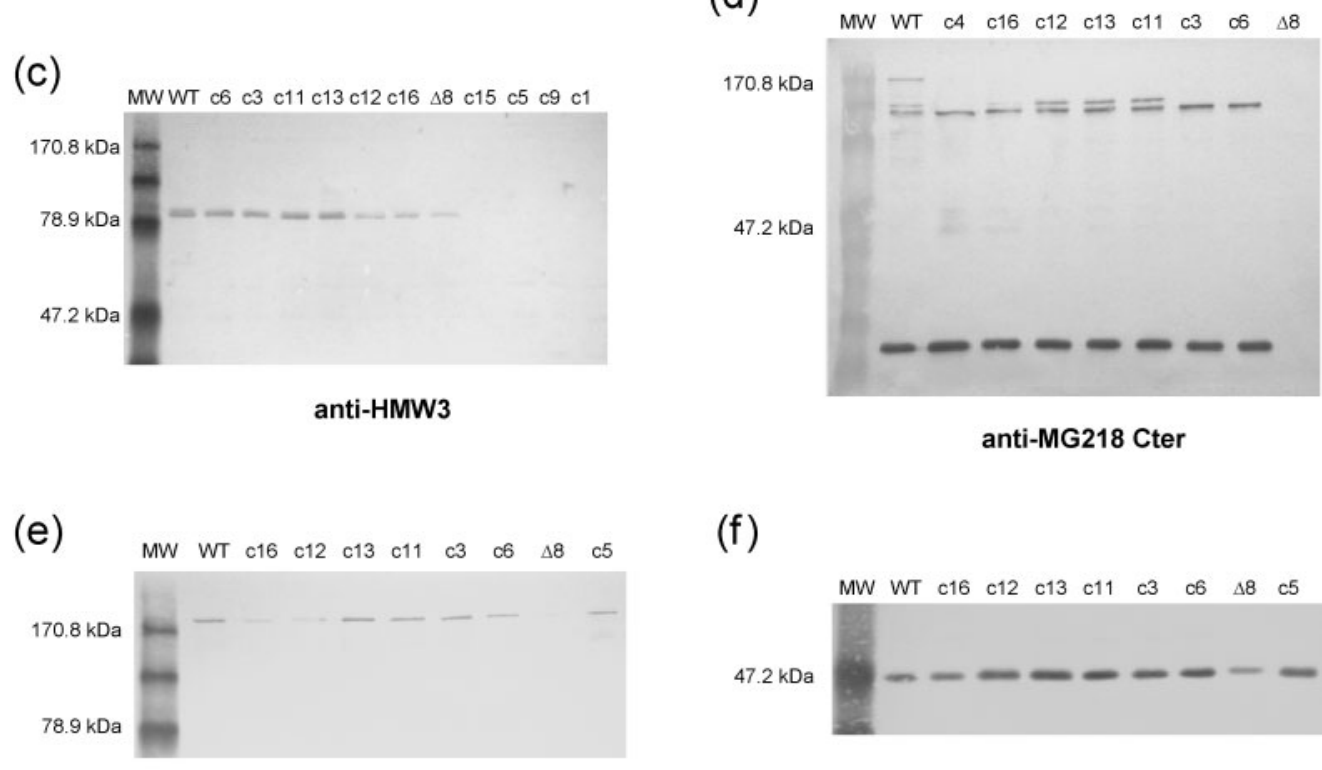

(f)

anti-HMW1

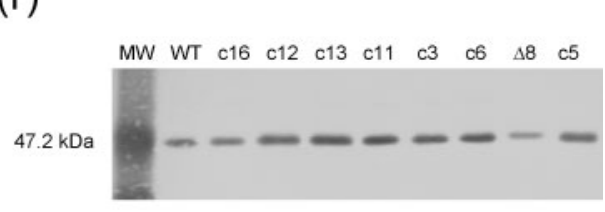

anti-P41

Fig. 4. Protein profile of the wild-type strain (WT) and different mutant strains analysed by $8 \%$ SDS-PAGE (a) or Western blotting using antiserum to MG218 N-terminal region (b), HMW3 (c), MG218 C-terminal region (d), HMW1 (e) or P41 proteins (f). MW, size markers. 
haemadsorption activity displayed by the two mg218mutants with severe SAD was similar to the activity observed for $\Delta \mathrm{mg} 218$ mutants (data not shown). All data regarding mg218 mutants with severe SAD obtained in this study are similar to the data obtained for $\Delta \mathrm{mg} 218$ mutants and will not be addressed further in this manuscript.

\section{Cell morphology}

Scanning electron microscopy analyses revealed that cells from mg218 ${ }^{-}$mutants with moderate SAD (Fig. 5b) and mg317 ${ }^{-}$mutants (Fig. 5c) exhibit a similar morphology, characterized by the presence of a short TO. The thin neck that joins the cell body and the TB in the wild-type cells (Fig. 5a, arrows) is less apparent in these mutants. Interestingly, most of the cells from these mutants show long tails at the pole opposite to the TO. These long tails often join cells oriented in opposite directions. Among the population of $\Delta \mathrm{mg} 218$ mutants (Fig. $5 \mathrm{~d}$, e), we observed rounded cells, reminiscent of those described previously for $\Delta \mathrm{mg} 191$ and $\Delta \mathrm{mg} 192$ mutants (Burgos et al., 2006), as well as elongated cells. However, the most remarkable feature of $\Delta \mathrm{mg} 218$ mutants is the presence of rounded bodies, two or threefold larger than a single wild-type cell, suggesting the existence of strong deficiencies in cell division.

\section{Ultrastructural analyses}

TOs were easily recognized in cells from the wild-type strain and they were defined by a long EDC $(214 \pm 7 \mathrm{~nm})$ with a characteristic curvature at the proximal end (Fig. 6a). In agreement with previous work (Henderson \& Jensen, 2006), some views revealed the origin of two independent rods at the proximal end of the EDC (Fig. 6a, numbers 1 and 2). In addition, a slightly electron-dense material that separates the cytoplasm from the proximal end of the EDC was observed (Fig. 6a, arrows). The distal end of the EDC showed the thicker region currently known as the TB. Notably, some transverse sections of the TB revealed the presence of several discrete highly electron-dense rounded particles (Fig. 6a, asterisk; see also Supplementary Fig. S1, available with the online version of this paper). Since the overall structure found in the M. genitalium TO seems similar to that observed in the M. pneumoniae TO, we hypothesize that these rounded particles correspond to the arched path of discrete globular proteins (Fig. 7b, component C) recently identified in $M$. pneumoniae by cryo-electron tomography (Henderson \& Jensen, 2006).

Cells from mg $218^{-}$mutants with moderate SAD showed TOs with EDCs that were shorter $(138 \pm 10 \mathrm{~nm})$ than those of the wild-type strain (Fig. 6b). TBs were still present and some sections retained the highly electron-dense rounded particles described for the wild-type strain. In contrast, these electron-dense particles were not observed in preparations from mg317 mutants. Most of the EDCs from $\mathrm{mg} 317^{-}$mutants were devoid of a clear TB, and the distal end of the EDC often appeared unconnected to the cell membrane (Fig. 6c). Interestingly, we also observed electron-dense structures embedded in the cytoplasm, not
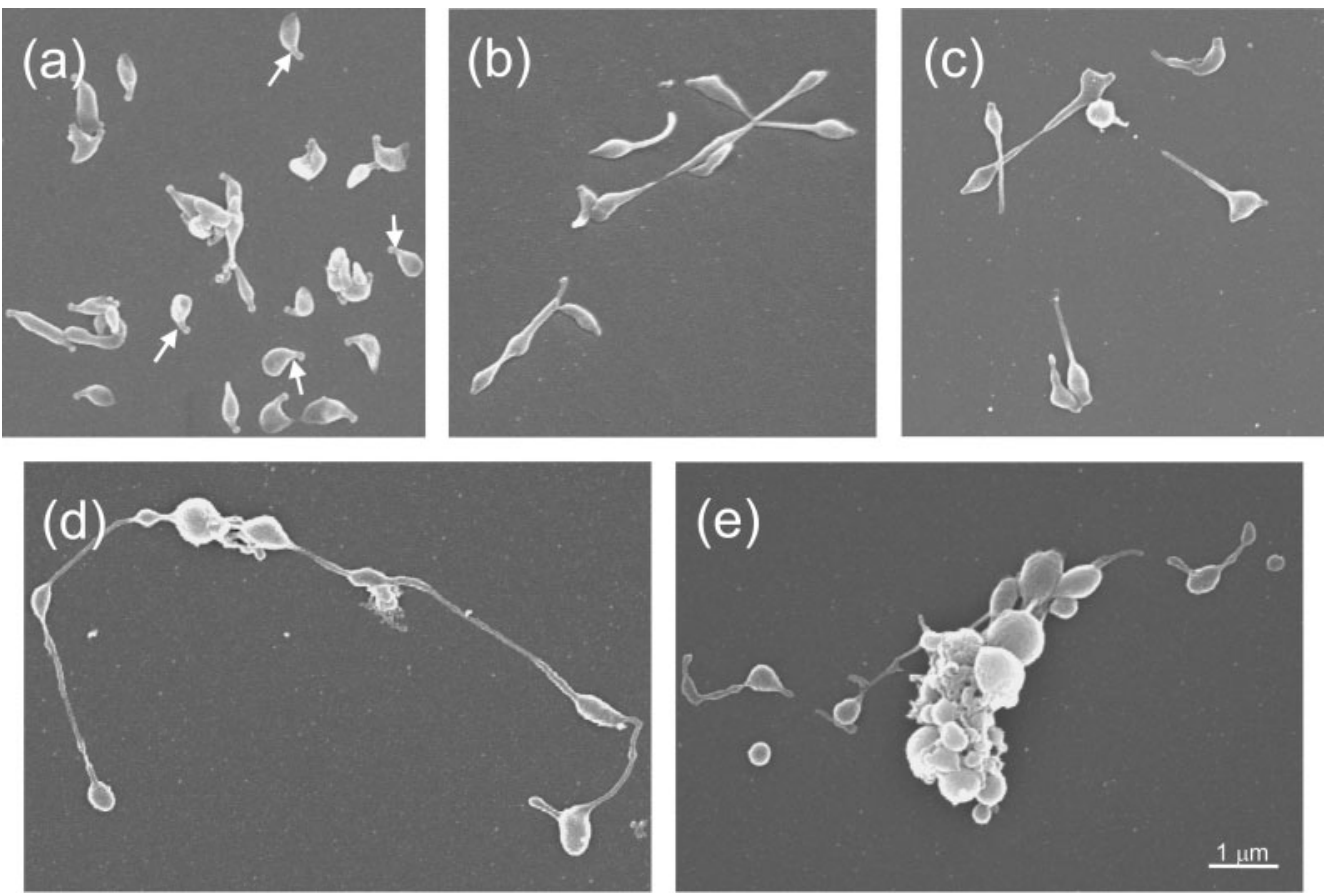

Fig. 5. Scanning electron microscopy of the wild-type strain (a), mg218 $8^{-}$mutant c6 (b), mg $317^{-}$mutant c5 (c) and a $\Delta$ mg 218 mutant ( $d$ and $e$ ). Arrows show the neck that joins the cell body and the TB in the wild-type strain. 

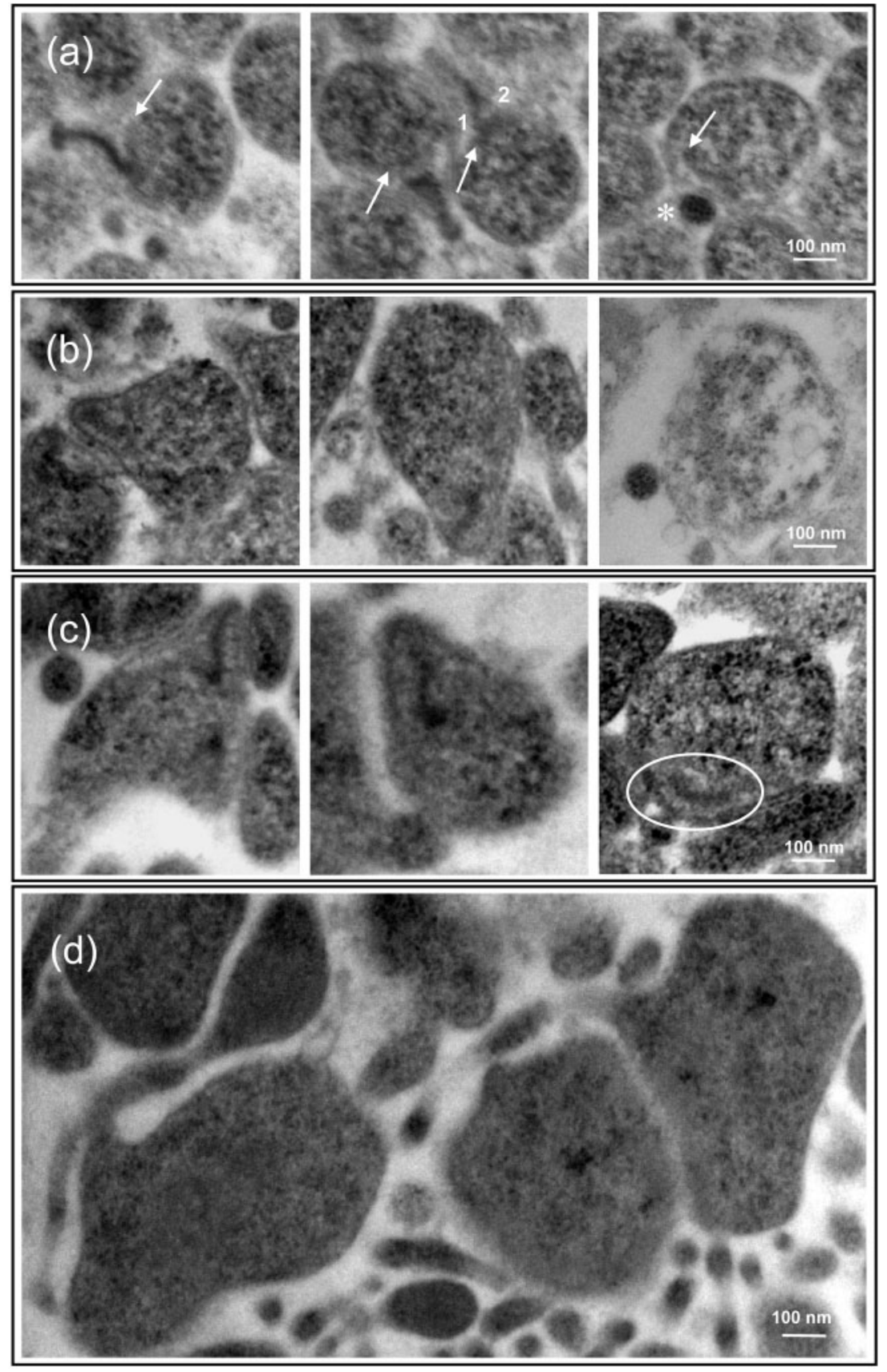

Fig. 6. Transmission electron microscopy of the wild-type strain (a), mg218 ${ }^{-}$mutant c6 (b), mg317- mutant c5 (c) and a $\Delta$ mg 218 mutant (d). The presence of two independent rods in the EDC is indicated by the numbers 1 and 2 . Arrows indicate the slightly electron-dense material that separates the cytoplasm from the EDC. The asterisk highlights the presence of rounded particles in transverse sections of the TB. The circle in the right panel of (c) draws attention to an EDC free in the cytoplasm and not associated with any membrane protrusion.

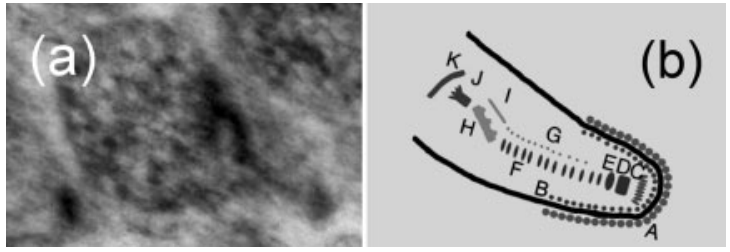

Fig. 7. Overall structure of $M$. genitalium TO (a) and schematic representation of the attachment organelle of $M$. pneumoniae with components labelled (b); modified from Henderson \& Jensen (2006). associated with any membrane protrusion (Fig. 6c). These structures resemble bona fide EDCs that appear to be free in the cytoplasm, since the tip structure could not be identified in such cells. These observations suggest that the distal end of the EDC is not attached tightly to the cell membrane in these mutants. In contrast, EDCs could not be identified in cells from $\Delta \mathrm{mg} 218$ mutants (Fig. $6 \mathrm{~d}$ ). In accordance with the images obtained by scanning electron microscopy, cell bodies from $\Delta \mathrm{mg} 218$ mutants were larger than those observed for the wild-type strain. Finally, many cells from all the mutants analysed in this study displayed long tails with alternating electron-dense and electron- 
transparent regions, as could be seen for $\Delta \mathrm{mg} 218$ mutants (Fig. 6d).

\section{DISCUSSION}

In the current study, we have found that transposon disruption of $m g 218$ or $m g 317$ genes does not completely eliminate the ability of the respective mutant cells to attach to solid surfaces, a feature that is thought to be essential for mycoplasma locomotion in vivo. The examination of $\mathrm{mg} 218^{-}$ and $\mathrm{mg} 317^{-}$mutants reveals a significant reduction in their number of motile cells and their gliding speed, in addition to the presence of anomalous TOs. This fact suggests that the involvement of MG218 and MG317 proteins in the gliding mechanics is a direct consequence of their respective roles in TO organization. However, we cannot discard the possibility that the reduced levels of MG386, P140 and P110 proteins, previously linked to mycoplasma gliding motility (Hasselbring et al., 2006; Pich et al., 2006a; Seto et al., 2005), could also contribute to the reduced locomotion observed in the $\mathrm{mg} 218^{-}$and $\mathrm{mg} 317^{-}$mutant cells.

The two different phenotypes observed among mg $218^{-}$ mutants are well correlated with the presence of $\mathrm{N}$ terminal MG218 truncated fragments. Supporting this information, we identified the presence of $\mathrm{N}$-terminal MG218 derivatives in all mg218 ${ }^{-}$mutants with moderate SAD. These MG218 truncated forms seem to be responsible for the EDC formation and the intermediate cytadherence activity displayed by this group of $\mathrm{mg} 218^{-}$mutants. The stability of the N-terminal MG218 derivatives with lengths ranging from 577 to 799 amino acids is interesting, because it has been reported that N-terminal HMW2 derivatives smaller than 1040 amino acids are unstable in $M$. pneumoniae (Balish et al., 2003a). Moreover, in contrast to M. pneumoniae hmw2 transposon mutants (Hedreyda \& Krause, 1995), N-terminal MG218 derivatives are not only stable but they are still functional in $M$. genitalium. Accordingly, the shorter EDCs observed in $\mathrm{mg} 218^{-}$ mutants with moderate SAD probably reflect the truncated nature of the N-terminal MG218 derivatives. Thereby, our results provide experimental evidence that the length of the EDC corresponds to the length of MG218 modelled as a fully extended $\alpha$-helix, as has been addressed previously for M. pneumoniae HMW2 (Balish et al., 2003b). Interestingly, we also identified the existence of multiple C-terminal MG218 derivatives in all $\mathrm{mg} 218^{-}$mutants as well as in the wild-type strain. The presence of these internal products in mg218 ${ }^{-}$mutants rules out the possibility that they could arise from the proteolytic processing of full-length MG218 protein and suggests the existence of additional transcriptional and translational start sites in the mg218 coding region. Two different ATG codons, encoding methionines 804 and 822 of MG218 protein, could also function as internal start codons, giving rise to polypeptides with a size compatible with the 118 and $115 \mathrm{kDa}$ C-terminal MG218 derivatives identified in this work (see Fig. $4 \mathrm{~d}$ and Supplementary Table S1, available with the online version of this paper). The function of these MG218 derivatives, not identified in $M$. pneumoniae, is still unknown. In contrast, the $25 \mathrm{kDa}$ C-terminal MG218 derivative, which could arise from the translation of MG218 starting at either methionine 1574 or 1615 (Supplementary Table S1), is probably the orthologue of $M$. pneumoniae P28 protein (Krause et al., 1997). The presence of this small derivative in both mycoplasma species suggests that it plays a significant biological function that still remains unknown. Thus, further work is needed to unravel the significance of the different C-terminal derivatives found in M. genitalium. On the other hand, it is noteworthy that $\Delta \mathrm{mg} 218$ mutants, lacking such C-terminal MG218 derivatives, exhibit a phenotype similar to that of $\mathrm{mg} 218^{-}$mutants with severe SAD. In addition, these C-terminal derivatives do not seem to be involved in the stabilization of MG312 and MG317 proteins, because MG312, and to a lesser extent MG317, are unstable in $\mathrm{mg} 218^{-}$mutants with severe SAD. Thus, it seems that the stability of MG312 and MG317 depends on the expression of the MG218 N-terminal region, in particular of the short stretch located between amino acids 577 and 648 (the length of the N-terminal MG218 derivatives in mutants $\mathrm{c} 12$ and $\mathrm{cl3}$ ). Altogether, these observations indicate that the MG218 N-terminal region can fulfil many of the roles currently associated with the expression of a full-length MG218 protein, a result that is in acute contrast to that observed in M. pneumoniae (Balish et al., 2003a).

Electron microscopy analyses reveal that MG317 protein is required to build up a standard TB. Since many cells from $m g 317$ transposon mutants show EDC free in the cytoplasm, the TB seems to be involved in the attachment of the distal end of the EDC to the inner surface of the plasma membrane. It is tempting to speculate that MG317 is involved in the attachment of the EDC to the intracellular domains of P140/ P110 complexes present at the terminal tip membrane. To fulfil such a possible function, MG317 should be localized in the vicinity of the TB in M. genitalium, which is in agreement with the localization of HMW3 in M. pneumoniae revealed by immunoelectron microscopy (Stevens \& Krause, 1992). In our micrographs, the chevron-like structures previously observed in a M. pneumoniae hmw3 mutant (Willby \& Krause, 2002) could not be identified. Regardless of this, the intermediate cytadherence displayed by our $\mathrm{mg} 317^{-}$ mutants is in accordance with the phenotype described for the M. pneumoniae hmw3 mutant (Willby \& Krause, 2002). Thus, the partial cytadherence activity displayed by $\mathrm{mg} 317^{-}$ and $\mathrm{mg} 218^{-}$mutants provides an explanation for the previous failure to isolate $M$. genitalium haemadsorptionnegative mutants showing transposon insertions in these two cytadherence-related genes (Reddy et al., 1996). Interestingly, a similar situation could be hampering the isolation of Mycoplasma gallisepticum haemadsorption-negative mutants bearing transposon insertion in genes different from gapA and crmA (Mudahi-Orenstein et al., 2003). Therefore, screening for colonies exhibiting a compact morphology could provide a direct way to isolate these mutants. 
As stated previously, all the mutants isolated in this study show reduced levels of P140 and P110 proteins, which are involved in the maintenance of M. genitalium cytadherence (Burgos et al., 2006). This result indicates that beyond the mutual interdependence of P140 and P110, demonstrated previously in $\Delta \mathrm{mg} 191$ and $\Delta \mathrm{mg} 192$ mutants (Burgos et al., 2006), the levels of these two proteins seem to be also coordinately regulated in M. genitalium. In addition, the reduced levels of P140 and P110 also provide an explanation for the reduced haemadsorption activity exhibited by $\mathrm{mg} 218^{-}$and $\mathrm{mg} 317^{-}$mutants. However, additional factors are needed to explain the reduced cytadherence observed in $\mathrm{mg} 218^{-}$mutants with severe SAD. Probably, the absence of a TO in these mutants is also hampering the correct localization of these two adhesins in the outer side of the cell membrane. Notably, a previous study in which M. genitalium mg218 was disrupted through homologous recombination allowed the isolation of some mutants (designated JB1 mutants) displaying a cytadherence-negative phenotype (Dhandayuthapani et al., 1999). JB1 mutants exhibited a dramatic instability of P140 and P110 that might also explain the complete loss of cytadherence of these mutants. In contrast, P140 and P110 are still found in all the $\Delta \mathrm{mg} 218$ mutants isolated in our study, although at reduced levels. At the present time, the origin of the different behaviour of P140 and P110 in JB1 and $\Delta \mathrm{mg} 218$ mutants is unclear.

Finally, most of the cells from the $\mathrm{mg} 218^{-}$and $\mathrm{mg} 317^{-}$ mutants showed thin filaments similar to those described previously for $m g 386$ and $m g 200$ gliding-deficient mutants (Pich et al., 2006a). Scanning electron microscopy images from $\mathrm{mg} 218^{-}$and $\mathrm{mg} 317^{-}$mutants also revealed that cells coupled by these filaments are always oriented in opposite directions, a configuration characteristic of dividing mycoplasma cells. Altogether, these observations suggest that cytokinesis is impaired in M. genitalium mutants with gliding motility defects. In addition, locomotion clearly contributes to cell dispersion in adherent mycoplasmas, a fact that is supported by the formation of large cell aggregates in liquid cultures of mutants with gliding deficiencies (Pich et al., 2006a). The dramatic consequences for cell division, probably magnified by the complete loss of the TO, are particularly evident in $\Delta$ mg218 mutants. However, the large cell bodies observed in $\Delta \mathrm{mg} 218$ mutants may reveal an additional role for MG218. Interestingly, further investigation of conserved domains within MG218 (Marchler-Bauer \& Bryant, 2004) shows the presence of two independent but overlapping SMC domains (Structural Maintenance of Chromosomes; COG1196). Proteins that contain SMC-domains have been shown to participate in many aspects of higher-order chromosome organization. Like SMC proteins, MG218 is predicted to form large coiled-coils that are often involved in protein-DNA interactions (Akhmedov et al., 1999). Additional analyses are needed to determine whether DNA is present in the TO and whether MG218 protein is also involved in chromosome dynamics of M. genitalium.

\section{ACKNOWLEDGEMENTS}

This work was supported by grant BFU2004-06377-C02-01 to E. Q. R. B. acknowledges an FPU predoctoral fellowship from Ministerio de Educación y Ciencia and O.Q.P. acknowledges a predoctoral fellowship from CeRBa (Centre de Referència en Biotecnologia). We thank Dr D. C. Krause, University of Georgia, Athens, GA, USA, for providing HMW1, HMW3 and P41 antisera. We appreciate the work of the whole staff of the Servei de Microscòpia (UAB) in processing electron microscopy samples. We are indebted to Alejandro Sánchez-Chardi and Francisca Cardoso for their valuable advice on performing transmission and scanning electron microscopy analyses, respectively. Finally, we are grateful to Dr J. B. Baseman for his helpful comments when reading the manuscript.

\section{REFERENCES}

Akhmedov, A. T., Gross, B. \& Jessberger, R. (1999). Mammalian SMC3 C-terminal and coiled-coil protein domains specifically bind palindromic DNA, do not block DNA ends, and prevent DNA bending. J Biol Chem 274, 38216-38224.

Balish, M. F., Ross, S. M., Fisseha, M. \& Krause, D. C. (2003a). Deletion analysis identifies key functional domains of the cytadherence-associated protein HMW2 of Mycoplasma pneumoniae. Mol Microbiol 50, 1507-1516.

Balish, M. F., Santurri, R. T., Ricci, A. M., Lee, K. K. \& Krause, D. C. (2003b). Localization of Mycoplasma pneumoniae cytadherenceassociated protein HMW2 by fusion with green fluorescent protein: implications for attachment organelle structure. Mol Microbiol 47, 49-60.

Baseman, J. B. \& Tully, J. G. (1997). Mycoplasmas: sophisticated, reemerging, and burdened by their notoriety. Emerg Infect Dis 3, 21-32.

Baseman, J. B., Dallo, S. F., Tully, J. G. \& Rose, D. L. (1988). Isolation and characterization of Mycoplasma genitalium strains from the human respiratory tract. J Clin Microbiol 26, 2266-2269.

Burgos, R., Pich, O. Q., Ferrer-Navarro, M., Baseman, J. B., Querol, E. \& Pinol, J. (2006). Mycoplasma genitalium P140 and P110 cytadhesins are reciprocally stabilized and required for cell adhesion and terminalorganelle development. J Bacteriol 188, 8627-8637.

Burgos, R., Pich, O. Q., Querol, E. \& Pinol, J. (2007). Functional analysis of the Mycoplasma genitalium MG312 protein reveals a specific requirement of the MG312 N-terminal domain for gliding motility. J Bacteriol 189, 7014-7023.

Dhandayuthapani, S., Rasmussen, W. G. \& Baseman, J. B. (1999). Disruption of gene $m g 218$ of Mycoplasma genitalium through homologous recombination leads to an adherence-deficient phenotype. Proc Natl Acad Sci U S A 96, 5227-5232.

Fraser, C. M., Gocayne, J. D., White, O., Adams, M. D., Clayton, R. A., Fleischmann, R. D., Bult, C. J., Kerlavage, A. R., Sutton, G. \& other authors (1995). The minimal gene complement of Mycoplasma genitalium. Science 270, 397-403.

Gobel, U., Speth, V. \& Bredt, W. (1981). Filamentous structures in adherent Mycoplasma pneumoniae cells treated with nonionic detergents. J Cell Biol 91, 537-543.

Hasselbring, B. M. \& Krause, D. C. (2007). Cytoskeletal protein P41 is required to anchor the terminal organelle of the wall-less prokaryote Mycoplasma pneumoniae. Mol Microbiol 63, 44-53.

Hasselbring, B. M., Jordan, J. L. \& Krause, D. C. (2005). Mutant analysis reveals a specific requirement for protein P30 in Mycoplasma pneumoniae gliding motility. J Bacteriol 187, 6281-6289. 
Hasselbring, B. M., Page, C. A., Sheppard, E. S. \& Krause, D. C. (2006). Transposon mutagenesis identifies genes associated with Mycoplasma pneumoniae gliding motility. J Bacteriol 188, 6335-6345.

Hedreyda, C. T. \& Krause, D. C. (1995). Identification of a possible cytadherence regulatory locus in Mycoplasma pneumoniae. Infect Immun 63, 3479-3483.

Henderson, G. P. \& Jensen, G. J. (2006). Three-dimensional structure of Mycoplasma pneumoniae's attachment organelle and a model for its role in gliding motility. Mol Microbiol 60, 376-385.

Krause, D. C. (1996). Mycoplasma pneumoniae cytadherence: unravelling the tie that binds. Mol Microbiol 20, 247-253.

Krause, D. C. \& Balish, M. F. (2004). Cellular engineering in a minimal microbe: structure and assembly of the terminal organelle of Mycoplasma pneumoniae. Mol Microbiol 51, 917-924.

Krause, D. C., Proft, T., Hedreyda, C. T., Hilbert, H., Plagens, H. \& Herrmann, R. (1997). Transposon mutagenesis reinforces the correlation between Mycoplasma pneumoniae cytoskeletal protein HMW2 and cytadherence. J Bacteriol 179, 2668-2677.

Lluch-Senar, M., Vallmitjana, M., Querol, E. \& Piñol, J. (2007). A new promoterless reporter vector reveals antisense transcription in Mycoplasma genitalium. Microbiology 153, 2743-2752.

Marchler-Bauer, A. \& Bryant, S. H. (2004). CD-Search: protein domain annotations on the fly. Nucleic Acids Res 32, W327-W331.

Meng, K. E. \& Pfister, R. M. (1980). Intracellular structures of Mycoplasma pneumoniae revealed after membrane removal. J Bacteriol 144, 390-399.

Mernaugh, G. R., Dallo, S. F., Holt, S. C. \& Baseman, J. B. (1993). Properties of adhering and nonadhering populations of Mycoplasma genitalium. Clin Infect Dis 17 (Suppl. 1), S69-S78.

Mudahi-Orenstein, S., Levisohn, S., Geary, S. J. \& Yogev, D. (2003). Cytadherence deficient mutants of Mycoplasma gallisepticum generated by transposon mutagenesis. Infect Immun 71, 3812-3820.

Perkins, D. N., Pappin, D. J., Creasy, D. M. \& Cottrell, J. S. (1999). Probability based protein identification by searching sequence databases using mass spectrometry data. Electrophoresis 20, 35513567.

Pich, O. Q., Burgos, R., Ferrer-Navarro, M., Querol, E. \& Pinol, J. (2006a). Mycoplasma genitalium $m g 200$ and $m g 386$ genes are involved in gliding motility but not in cytadherence. Mol Microbiol 60, 1509-1519.
Pich, O. Q., Burgos, R., Planell, R., Querol, E. \& Pinol, J. (2006b). Comparative analysis of antibiotic resistance gene markers in Mycoplasma genitalium: application to studies of the minimal gene complement. Microbiology 152, 519-527.

Radestock, U. \& Bredt, W. (1977). Motility of Mycoplasma pneumoniae. J Bacteriol 129, 1495-1501.

Reddy, S. P., Rasmussen, W. G. \& Baseman, J. B. (1996). Isolation and characterization of transposon Tn4001-generated, cytadherencedeficient transformants of Mycoplasma pneumoniae and Mycoplasma genitalium. FEMS Immunol Med Microbiol 15, 199-211.

Roberts, R. J. (2004). Identifying protein function - a call for community action. PLoS Biol 2, E42.

Seto, S., Kenri, T., Tomiyama, T. \& Miyata, M. (2005). Involvement of P1 adhesin in gliding motility of Mycoplasma pneumoniae as revealed by the inhibitory effects of antibody under optimized gliding conditions. J Bacteriol 187, 1875-1877.

Stevens, M. K. \& Krause, D. C. (1991). Localization of the Mycoplasma pneumoniae cytadherence-accessory proteins HMW1 and HMW4 in the cytoskeleton-like Triton shell. J Bacteriol 173, 1041-1050.

Stevens, M. K. \& Krause, D. C. (1992). Mycoplasma pneumoniae cytadherence phase-variable protein HMW3 is a component of the attachment organelle. J Bacteriol 174, 4265-4274.

Svenstrup, H. F., Jensen, J. S., Gevaert, K., Birkelund, S. \& Christiansen, G. (2006). Identification and characterization of immunogenic proteins of Mycoplasma genitalium. Clin Vaccine Immunol 13, 913-922.

Taylor-Robinson, D. \& Horner, P. J. (2001). The role of Mycoplasma genitalium in non-gonococcal urethritis. Sex Transm Infect 77, 229-231.

Tully, J. G., Rose, D. L., Whitcomb, R. F. \& Wenzel, R. P. (1979). Enhanced isolation of Mycoplasma pneumoniae from throat washings with a newly-modified culture medium. J Infect Dis 139, 478-482.

Tully, J. G., Taylor-Robinson, D., Cole, R. M. \& Rose, D. L. (1981). A newly discovered mycoplasma in the human urogenital tract. Lancet 1, 1288-1291.

Willby, M. J. \& Krause, D. C. (2002). Characterization of a Mycoplasma pneumoniae $h m w 3$ mutant: implications for attachment organelle assembly. J Bacteriol 184, 3061-3068.

Edited by: C. Citti 